\title{
La interoperabilidad tecnológica para una universidad inteligente
}

\section{Technological interoperability for a smart university}

CANEDO-ROMERO, Gerardo Enrique†, CANEDO-MONTOYA, Gerardo Miguel y CANEDOMONTOYA, Enrique Daniel

\section{Universidad de Guanajuato, México.}

Universidad Politécnica de Juventino Rosas, México.

ID $1^{\text {er }}$ Autor: Gerardo Enrique, Canedo-Romero / ORC ID: 0000-0001-6624-185X, SNI CONACYT ID: 994269

ID $1^{\text {er }}$ Coautor: Gerardo Miguel, Canedo-Montoya / ORC ID: 0000-0002-4036-2394, SNI CONACYT ID: 994258

ID $2^{\text {do }}$ Coautor: Enrique Daniel, Canedo-Montoya / ORC ID: 0000-0002-8062-9567, SNI CONACYT ID: 520884

\section{Resumen}

Con la llegada de la sociedad del conocimiento las organizaciones inteligentes están modificando las actividades del ser humano. La universidad no está ajena a su transformación. La universidad inteligente proporcionara servicios de educación superior con una cobertura mayor y por lo tanto a menor costo. Este trabajo propone un modelo para la interoperabilidad en los sistemas de información y tecnologías de la información en una universidad inteligente.

Universidad Inteligente, Modelo innovador, Interoperabilidad

\section{Abstract}

In this work of diagnostic nature, addresses aspects of the With the advent of the knowledge society, intelligent organizations are changing human activities. The university is no stranger to its transformation. The smart university will provide higher education services with higher coverage and therefore at lower cost. This work proposes a model for interoperability in information systems and information technologies in an intelligent university.

Intelligent University, Innovative model, Interoperability

Citación: CANEDO-ROMERO, Gerardo Enrique, CANEDO-MONTOYA, Gerardo Miguel y CANEDO-MONTOYA, Enrique Daniel. La interoperabilidad tecnológica para una universidad inteligente. Revista de Educación Técnica. 2020. 411:8-11. 


\section{Introducción}

La sociedad del conocimiento está caracterizada por la interacción y la interoperabilidad de sus usuarios y de las tecnologías respectivamente.

En este trabajo se propone un modelo para una universidad inteligente de interoperabilidad de los procesos mediante la automatización en los sistemas de información y de las operaciones ejecutadas por las tecnologías de la información.

En este trabajo se propone un modelo que aumente la cobertura y la matrícula para los sectores económicamente vulnerables.

\section{La organización inteligente}

Se puede describir el concepto de una organización inteligente de la siguiente manera [1]: la Organización Inteligente es una entidad que detecta la información de su entorno interno y de las tendencias de su entorno externo para la generación del conocimiento que permita la toma de decisiones a nivel estratégico para el cumplimiento de los objetivos de la organización.

Uno de los pilares de la organización inteligente es la innovación, desde la innovación organizacional pasando por la organización de sistemas de información y la innovación tecnológica.

La generación del conocimiento implica a la innovación inteligente en dos aspectos:

- Interacción. El paradigma innovador de las relaciones humanas con la automatización de los procesos.

- Interoperabilidad. La aplicación de la inteligencia artificial distribuida para la automatización de los procesos (ver Figura $1)$.

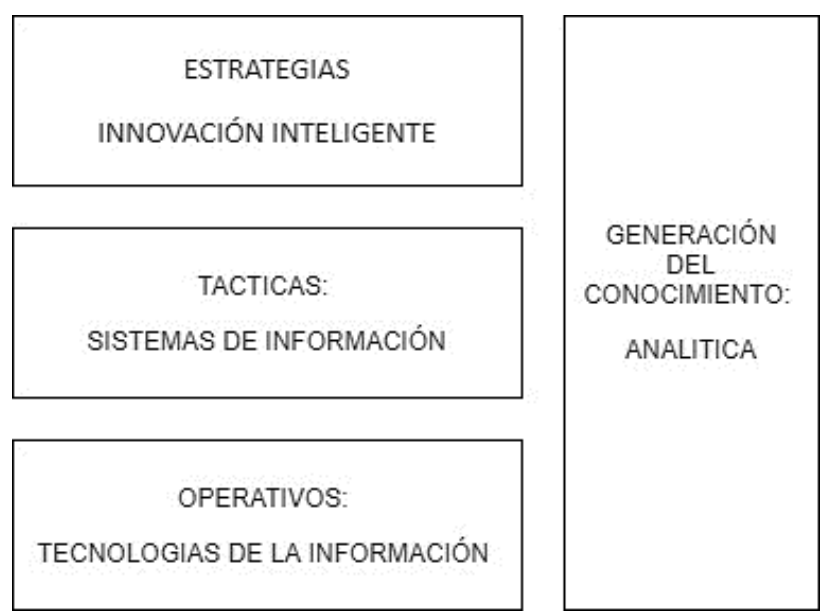

Figura 1 Arquitectura de una Organización Inteligente Fuente: elaboración propia [Draw.io]

\section{Modelos de universidad}

Históricamente la universidad ha tenido 4 modelos:

- Modelos clásicos: modelo francés y modelos Alemán. El modelo francés le dio importancia a la función sustantiva de la docencia relegando a la función sustantiva de la investigación. Este modelo se caracterizó por la formación teórica de los estudiantes. Su estructura curricular fue de planes de estudio rígidos y la modalidad escolarizada. El modelo Alemán se caracterizó por dar importancia a la función sustantiva de la investigación relegando a la docencia. La formación de los estudiantes se consideraba práctica. Su estructura curricular fue de planes de estudio rígidos y la modalidad escolarizada.

- Modelo Anglosajón. El modelo Anglosajón fue el primer modelo pensando en el estudiante con un compromiso entre las dos funciones sustantivas de docencia e investigación. Su estructura curricular fue de planes de estudio rígidos y la modalidad escolarizada. La flexibilidad de este modelo consiste en la adecuación de las cargas de trabajo de cada estudiante en lo individual.

- Modelo Digital. Partiendo del modelo anglosajón y de la llegada de internet el estudiante tiene posibilidades de estudiar algunos cursos bajo la modalidad a distancia. La estructura es rígida y la modalidad es escolarizada y a distancia. 
- Modelo Inteligente. La universidad inteligente está centrada en el estudiante con estructuras curriculares flexibles y con la impartición de cursos a distancia. Este modelo de universidad debe de contar con instrumentos de control para dar seguimiento a las cargas académicas de cada uno de sus estudiantes.

\section{Definición del problema}

Como se muestra en la figura, la universidad bajo el esquema digital, aún se encuentra en la etapa de maduración. Sin embargo, la universidad inteligente ya se encuentra en su etapa de innovación por lo que falta un modelo para la transición del modelo de la universidad digital al modelo de universidad inteligente (ver Figura 2).

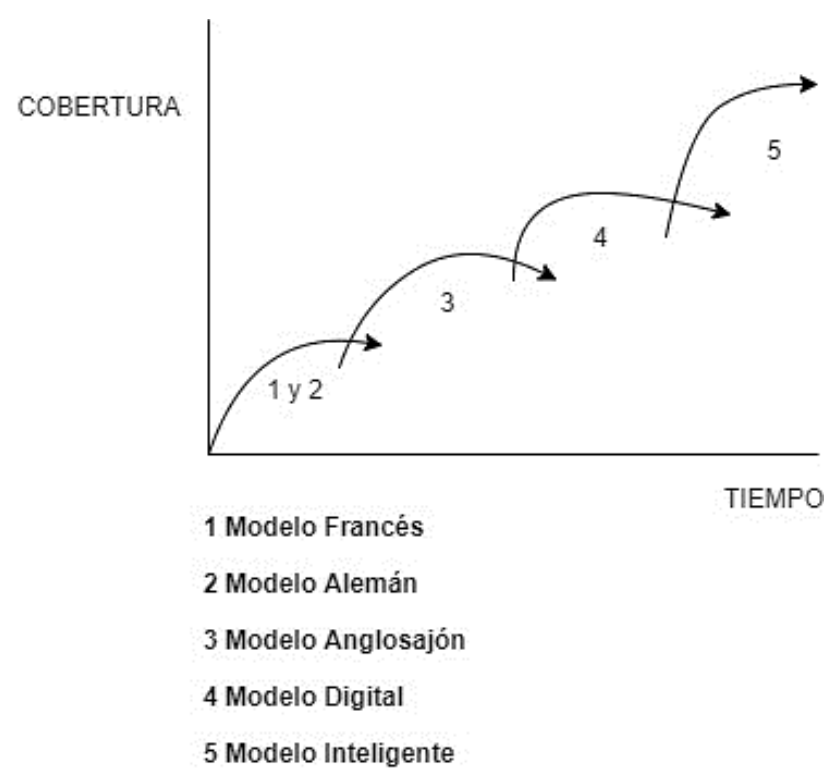

Figura 2 Modelos de Universidad

Fuente: elaboración propia [Draw.io]

Se han realizado esfuerzos para modelar una universidad inteligente [2, 3, 4] 1 Modelo Francés 2 Modelo Alemán 3 Modelo Anglosajón 4 Modelo Digital 5 Modelo Inteligente.

El presente trabajo propone un modelo para la interoperabilidad de las funciones sustantivas de la universidad inteligente en lo horizontal y en lo vertical.

\section{Modelo propuesto}

El modelo propuesto en el presente trabajo, parte de las siguientes premisas:
- A nivel estratégico el empleo de la innovación para el establecimiento de estructuras curriculares inteligentes $\mathrm{y}$ flexibles.

- Automatización de los procesos que soporten las funciones sustantivas de la universidad en lo estratégico, táctico y operacional, es decir, establecer la conectividad horizontal y la conectividad vertical para que los servicios operen de manera satisfactoria.

- La universidad inteligente debe de considerar en su plan estratégico a los sistemas y tecnologías de la información como pilares que soporten sus procesos de enseñanza y aprendizaje.

- Las herramientas que permitan una automatización eficiente de todos los procesos de la universidad inteligente son los sistemas multi agente $\mathrm{y}$ el internet de las cosas (Ver Figura 3).

\begin{tabular}{|c|c|c|}
\hline \multicolumn{3}{|c|}{ Estrategia inteligente } \\
\hline $\begin{array}{l}\stackrel{\gtrsim}{\overline{0}} \\
\text { 응 }\end{array}$ & \begin{tabular}{|c|c}
$\begin{array}{c}\text { Propuesta de Valor } \\
\text { Plan de Estudios Inteligente } \\
\text { Proceso Inteligente de Enseñanza- } \\
\text { Aprendizaje }\end{array}$ \\
$\begin{array}{c}\text { Usuarios } \\
\text { Alumnos, Profesores y de Apoyo }\end{array}$ \\
$\begin{array}{c}\text { Sistemas de información } \\
\text { Inteligencia Artificial Distribuida }\end{array}$ \\
Tecnologías de la información \\
Internet de las Cosas
\end{tabular} & $\begin{array}{c}\text { Gestor del } \\
\text { conocimiento } \\
\text { Visualizadores } \\
\text { Big Data } \\
\text { Minería de } \\
\text { Datos } \\
\text { Almacén de } \\
\text { Datos }\end{array}$ \\
\hline
\end{tabular}

Figura 3 Modelo propuesto para Universidad Inteligente Fuente: elaboración propia [Draw.io]

\section{Conclusiones}

El modelo propuesto para la interoperabilidad tecnológica para una universidad inteligente cumple con las premisas establecidas en su desarrollo.

Como trabajo futuro se seleccionará un marco de trabajo para la caracterización de la universidad inteligente por medio de sistemas multi agente y a su vez se seleccionará un sistema de simulación para comprobar la verificación y validación de la universidad inteligente con el modelo propuesto. 


\section{Referencias}

[1] Choo C.W., La Organización Inteligente: El empleo de la información para dar significado, crear conocimiento y tomar decisiones, Oxford University Press, 1999.

[2] Amrollahi A., and B. Rowlands, (2018), OSPM: A design methodology for open strategic planning, Information \& Management, ELSEVIER.

[3] Ghemawat P., (2017), Strategies for Higher Education in the Digital Age, management education, SAGE.

[4] Yu X., and B. Zhang, (2018), Obtaining advantages from technology revolution: A patent roadmap for competition análisis and strategy planning, Technological Forecasting \& Social Change, ELSEVIER. 